\title{
Multivariate EWMA Control Chart for Means of Multiple Quality Variableswith Two Sampling Intervals
}

\author{
Duk-Joon Chang ${ }^{1}$ and Sunyeong $\mathrm{Heo}^{2 \dagger}$
}

\begin{abstract}
Because of the equivalence between control chart procedures and hypothesis testing, we propose to use likelihood ratio test (LRT) statistic $Z_{i}^{2}$ as the multivariate control statistic for simultaneous monitoring means of the multivariate normal process. Properties and comparisons of the proposed control charts are explored and conducted for matched fixed sampling interval (FSI) and variable sampling interval (VSI) with two sampling interval charts. The result of numerical comparisons shows that EWMA chart with two sampling interval procedure is more efficient than the corresponding FSI chart for small or moderate changes. When large shift of the process has occurred, we also found that Shewhart chart is more efficient than EWMA chart.
\end{abstract}

Key words : Signal, False Alarm Rate, Average Time to Signal

\section{Introduction}

Monitoring a production process over a time using a control chart allows quick detection of unusual causes of variation. And, statistical process control has been widely used for monitoring process parameters in industry. Therefore, ability of a control chart to detect process changes is determined by the length of time required for the chart to signal when the process is out-of-control state while producing few false alarms.

Since Shewhart introduced the control chart technique in 1924, control procedures have found widespread application in improving the quality of production processes. The Shewhart control chart is simple to understand and easy to construct. But the basic Shewhart chart uses only the information in the current sample and is thus relatively inefficient in detecting small or moderate changes in the process. In other words, the main disadvantage of the Shewhart chart is that it uses only the information from the last sample and is insensitive to small or moderate shifts in

${ }^{1}$ Department of statistics, Changwon National University, Changwon 641-773, Korea

${ }^{2}$ Department of statistics, Changwon National University, Changwon 641-773, Korea

${ }^{\dagger}$ Corresponding author: syheo@changwon.ac.kr

(Received : June 8, 2012, Revised : June 20, 2012,

Accepted : June 24, 2012) the process.

Exponentially weighted moving average (EWMA) control chart was first introduced by Roberts ${ }^{[1]}$. Like Shewhart control chart, an EWMA chart is easy to implement and interpret the process. In the EWMA scheme, the more recent observations are assigned more weights and the older observations are assigned less weights. Roberts showed that the EWMA chart is efficient in detecting small shifts in the process but is not efficient at large shifts. An EWMA has alternately been referred to as a geometric moving average because a function of current sample statistic $Z_{i}$ can be equivalently written as a moving average of the current and past samples as

$$
Z_{i}=(1-\lambda)^{i} Z_{0}+\lambda \sum_{j=0}^{i-1}(1-\lambda)^{j} Y_{i-j}
$$

$i=1,2, \ldots$ where $\lambda(0<\lambda \leq 1)$ is a smoothing constant.

One traditional practice in using a control chart is to take samples from the process at FSI and properties of control charts have been developed when the sampling interval between samples is fixed. The basic idea of VSI control chart is that the time interval should be short if there is some indication of a process change and should be long if there is no indication of a process change. VSI procedures were first investigated by Arnold ${ }^{[2]}$. Extension of Arnold's work was presented by Smeach 
and Jernigan ${ }^{[3]}$. They derived equations for the variance of the sample size and approximation schemes that could enhance the implementation of this sampling procedure.

Reynolds and Arnold ${ }^{[4]}$ developed general expressions for properties of a VSI Shewhart chart such as the average time to signal and the average number of samples to signal when the process is in-control or out-ofcontrol. Amin ${ }^{[5]}$ and Reynolds et al. ${ }^{[6]}$ investigated VSI CUSUM charts, and Cui and Reynolds investigated VSI Shewhart $\bar{X}$ charts with runs rules. Vargas et al. ${ }^{[7]}$ concluded the properties of EWMA schemes are similar to those of CUSUM schemes in terms of time to signal. Zou and Tsung ${ }^{[8]}$ investigated likelihood ratio- based distribution-free EWMA control charts.

In this paper, we investigate the properties and performances of multivariate EWMA charts for monitoring means of multiple quality variables simultaneously in terms of ANSS, ATS, ANSW, ASI and P (switch). The numerical performances of the proposed multivariate Shewhart and EWMA charts are obtained by markov chain method or simulation.

\section{Results and Discussion}

\subsection{Multivariate Shewhart Chart}

In many industrial quality control procedure, the quality of a product is usually characterized by joint levels of several correlated quality characteristics rather than a single characteristics. To monitor several correlated quality variables simultaneously, we assume that the quality of a product has $p(p \geq 2)$ quality variables represented by the random vector $\underline{X}=\left(X_{1}, \ldots, X_{p}\right)^{\prime}$. Let us assume that successive observation vectors represent a random sample from a multivariate normal population $N_{p}\left(\underline{\mu}, \Sigma_{0}\right)$ with mean vector $\underline{\mu}$ and variance-covariance matrix $\Sigma$.

In this paper, we assume that $\underline{\theta}_{0}=\left(\mu_{0}, \Sigma_{0}\right)$ be the known target process values for $\underline{\theta}=\left(\underline{\mu}, \Sigma_{0}\right)$. We take a sequence of random vectors $X_{1}, X_{2}, \ldots$ where $\underline{X}_{i}=$ $\left(\underline{X}_{i 1}^{\prime}, \ldots, \underline{X}_{i n}^{\prime}\right)^{\prime}$ is a sample of observations at the sampling time $i(i=1,2, \ldots)$ and $\underline{X}_{i j}=\left(X_{i j 1}, X_{i j 2}, \ldots, X_{i j p}\right)^{\prime}$. Then the joint density function of all observations is given by

$$
\frac{1}{(2 \pi)^{n p / 2}|\Sigma|^{n / 2}} \exp \left[-\frac{1}{2}(\underline{X}-\underline{\mu})^{\prime} \Sigma^{-1}(\underline{X}-\underline{\mu})\right] .
$$

Because a control chart can be viewed as repeated tests of significance, we can obtain multivariate control statistic by using the LRT statistic for testing $H_{0}: \underline{\mu}=\underline{\mu}_{0}$ vs $H_{1}: \underline{\mu} \neq \underline{\mu}_{0}$ where $\Sigma_{0}$ is known.

Likelihood ratio $\lambda$ at the $i$ th sample can be expressed as

$$
\lambda=\exp \left[-\frac{n}{2}\left(\underline{X}_{i}-\underline{\mu}\right)^{\prime} \Sigma_{0}^{-1}\left(\underline{X}_{i}-\underline{\mu}\right)\right] .
$$

Let $Z_{i}^{2}$ be $-2 \ln \lambda$. Then

$$
Z_{i}^{2}=n\left(\underline{X}_{i}-\underline{\mu}\right)^{\prime} \Sigma_{0}^{-1}\left(\underline{X}_{i}-\underline{\mu}\right)
$$

Thus, the test statistic $Z_{i}^{2}$ under $H_{0}: \underline{\mu}=\underline{\mu}_{0}$ can be used as the control statistic for monitoring means of $p$ related quality variables.

A multivariate FSI Shewhart chart for means based on the LRT statistic $Z_{i}^{2}$ signals whenever

$$
Z_{i}^{2} \geq h_{S}
$$

For two sampling interval VSI Shewhart chart based on LRT statistic $Z_{i}^{2}$, suppose that the sampling interval ;

$$
\begin{aligned}
& d_{1} \text { is used when } Z_{i}^{2} \in\left(g_{S}, h_{S}\right], \\
& d_{2} \text { is used when } Z_{i}^{2} \in\left(0, g_{S}\right],
\end{aligned}
$$

where $g_{S} \leq h_{S}$ and $d_{1}<d_{2}$.

In FSI chart, the length of the sampling interval between sampling times is constant for all sampling occasion $i(i=1,2, \ldots)$. But for a VSI control chart, the sampling times are random variables and the sampling interval $t_{i+1}-t_{i}$ depends on the past sample informations of $\underline{X}_{1}, \underline{X}_{2}, \ldots \underline{X}_{i}$. In VSI chart, it is necessary to keep track of both ATS and ANSS. Following the definition of Reynolds et al., the number of samples to signal (NSS) is taken from the start of the process to the time when the chart signals and ANSS is the expected value of the NSS. Also, they defined that the time to signal (TS) is taken from the start of the process to the time when the chart signals and ATS is the expected value of the TS. One disadvantage of VSI scheme is that frequent switching between different sampling intervals requires more cost and effort to administer the process than corresponding FSI scheme.

When two sampling interval VSI procedure is used, let $\psi_{i}$ represent the expected number of samples before 
the signal that $d_{i}(i=1,2)$ is used and the sampling interval before the first sample $d_{0}$ is either $d_{1}$ or $d_{2}$, then

$$
A N N S S=1+\psi_{1}+\psi_{2}
$$

and

$$
A T S=d_{0}+d_{1} \psi_{1}+d_{2} \psi_{2}=d \cdot A S N N,
$$

where $d$ can be interpreted as the average sampling interval (ASI).

Because a VSI chart switches between different sampling intervals, properties such as the time required to signal will be more difficult to evaluate than for the corresponding FSI chart. In addition, it will be desirable to evaluate properties, such as the amount of switching done by the VSI chart, which are not issue in FSI charts.
Therefore, it is necessary to consider a new quantity which measures the frequency of switches in VSI control procedures. Define the average number of switches (ANSW) to be the expected value of the number of switches made from the start of the process until the chart signals. The ANSW can be obtained as follows

$$
A N S W=\left(A N S S-d_{0}\right) \cdot P(\text { switch })
$$

And, the probability of switch is given by

$$
P(\text { switch })=P\left(d_{1}\right) \cdot P\left(d_{2} \mid d_{1}\right)+P\left(d_{2}\right) \cdot P\left(d_{1} \mid d_{2}\right)
$$

where $P\left(d_{i}\right)$ is the probability of using sampling interval $d_{i}$, and $P\left(d_{i} \mid d_{j}\right)$ is the conditional probability of using sampling interval $d_{i}$ in the current sample given that the

Table 1. Performances of multivariate EWMA chart $(p=5, \lambda=0.1)$

\begin{tabular}{cccccccccc}
\hline & FSI & \multicolumn{3}{c}{ VSI $:\left(d_{1}, d_{2}\right)=(0.7,1.3)$} & \multicolumn{4}{c}{ VSI $:\left(d_{1}, d_{2}\right)=(0.1,1.9)$} \\
\cline { 2 - 10 } & ARL & ATS & ANSW & ASI & P(sw) & ATS & ANSW & ASI & P(sw) \\
\hline in-control & 370.40 & 370.40 & 51.81 & 1.00 & 0.14 & 370.40 & 51.81 & 1.00 & 0.14 \\
\hline$\tau=0.50$ & 194.11 & 187.43 & 25.83 & 0.97 & 0.13 & 164.26 & 25.83 & 0.85 & 0.13 \\
$\tau=1.00$ & 59.36 & 55.26 & 6.17 & 0.93 & 0.11 & 44.44 & 6.17 & 0.75 & 0.11 \\
$\tau=1.50$ & 25.56 & 24.98 & 2.75 & 0.98 & 0.11 & 23.34 & 2.75 & 0.92 & 0.11 \\
$\tau=2.00$ & 14.76 & 15.09 & 2.21 & 1.02 & 0.16 & 15.92 & 2.21 & 1.08 & 0.16 \\
$\tau=2.50$ & 9.92 & 10.35 & 2.05 & 1.04 & 0.23 & 11.71 & 2.05 & 1.18 & 0.23 \\
$\tau=3.00$ & 7.24 & 7.63 & 1.99 & 1.05 & 0.32 & 9.01 & 1.99 & 1.25 & 0.32 \\
$\tau=3.50$ & 5.58 & 5.88 & 1.94 & 1.05 & 0.42 & 7.19 & 1.94 & 1.29 & 0.43 \\
$\tau=4.00$ & 4.44 & 4.68 & 1.88 & 1.05 & 0.55 & 5.90 & 1.88 & 1.33 & 0.55 \\
$\tau=4.50$ & 3.66 & 3.84 & 1.79 & 1.05 & 0.68 & 4.97 & 1.79 & 1.36 & 0.68 \\
$\tau=5.00$ & 3.08 & 3.21 & 1.67 & 1.04 & 0.81 & 4.27 & 1.67 & 1.39 & 0.81 \\
\hline
\end{tabular}

Table 2. Performances of multivariate EWMA chart $(p=5, \lambda=0.3)$

\begin{tabular}{cccccccccc}
\hline & FSI & \multicolumn{3}{c}{ VSI $:\left(d_{1}, d_{2}\right)=(0.7,1.3)$} & \multicolumn{4}{c}{ VSI $:\left(d_{1}, d_{2}\right)=(0.1,1.9)$} \\
\cline { 2 - 10 } & ARL & ATS & ANSW & ASI & P(sw $)$ & ATS & ANSW & ASI & P(sw $)$ \\
\hline in-control & 370.40 & 370.40 & 92.66 & 1.00 & 0.25 & 370.40 & 92.66 & 1.00 & 0.25 \\
\hline 218.62 & 213.57 & 54.19 & 0.98 & 0.25 & 194.85 & 54.19 & 0.88 & 0.25 \\
& 70.51 & 63.04 & 15.50 & 0.89 & 0.22 & 44.66 & 15.50 & 0.63 & 0.22 \\
& 23.58 & 19.98 & 4.32 & 0.85 & 0.19 & 11.93 & 4.32 & 0.51 & 0.19 \\
& 10.30 & 8.91 & 2.28 & 0.87 & 0.25 & 6.31 & 2.28 & 0.61 & 0.24 \\
& 5.96 & 5.31 & 1.85 & 0.89 & 0.37 & 4.55 & 1.85 & 0.76 & 0.37 \\
& 4.04 & 3.66 & 1.60 & 0.91 & 0.53 & 3.59 & 1.60 & 0.89 & 0.53 \\
& 3.00 & 2.74 & 1.34 & 0.91 & 0.67 & 2.96 & 1.34 & 0.99 & 0.67 \\
& 2.37 & 2.16 & 1.09 & 0.91 & 0.80 & 2.53 & 1.09 & 1.06 & 0.79 \\
& 1.95 & 1.78 & 0.84 & 0.91 & 0.88 & 2.23 & 0.84 & 1.14 & 0.88 \\
& 1.66 & 1.51 & 0.62 & 0.91 & 0.95 & 2.05 & 0.62 & 1.24 & 0.95 \\
\hline
\end{tabular}


Table 3. Performances of multivariate Shewhart chart $(p=5)$

\begin{tabular}{cccccccccc}
\hline & FSI & \multicolumn{3}{c}{ VSI : $\left(d_{1}, d_{2}\right)=(0.7,1.3)$} & \multicolumn{3}{c}{ VSI : $\left(d_{1}, d_{2}\right)=(0.1,1.9)$} \\
\cline { 2 - 9 } & ARL & ATS & ANSW & ASI & P(sw $)$ & ATS & ANSW & ASI & P(sw $)$ \\
\hline in-control & 370.39 & 370.39 & 183.70 & 1.00 & 0.50 & 370.40 & 183.70 & 1.00 & 0.50 \\
\hline 259.43 & 254.99 & 127.80 & 0.98 & 0.49 & 246.12 & 127.80 & 0.95 & 0.49 \\
& 114.38 & 107.17 & 53.20 & 0.94 & 0.47 & 92.73 & 53.20 & 0.81 & 0.47 \\
& 44.42 & 38.95 & 17.09 & 0.88 & 0.39 & 28.02 & 17.09 & 0.63 & 0.39 \\
& 17.93 & 14.76 & 4.60 & 0.82 & 0.27 & 8.41 & 4.60 & 0.47 & 0.27 \\
& 8.07 & 6.40 & 1.04 & 0.79 & 0.15 & 3.07 & 1.04 & 0.38 & 0.15 \\
& 4.16 & 3.32 & 0.19 & 0.80 & 0.06 & 1.63 & 0.19 & 0.39 & 0.06 \\
& 2.48 & 2.06 & 0.03 & 0.83 & 0.02 & 1.22 & 0.03 & 0.49 & 0.02 \\
& 1.70 & 1.50 & 0.00 & 0.88 & 0.00 & 1.08 & 0.00 & 0.64 & 0.00 \\
& 1.32 & 1.23 & 0.00 & 0.93 & 0.00 & 1.04 & 0.00 & 0.78 & 0.00 \\
& 1.14 & 1.10 & 0.00 & 0.96 & 0.00 & 1.01 & 0.00 & 0.89 & 0.00 \\
\hline
\end{tabular}

sampling interval $d_{j}\left(d_{i} \neq d_{j}\right)$ was used in the previous sample. To quantify the amount of switching, average switching rate (ASWR) can be defined as

$$
A S W R=A N S W / A N S S .
$$

A low value of the ASWR will usually be desirable from the administrative point of view, but a value of the ASWR very close to zero may not be achievable in a chart that is responsive to changes in the process.

Since the statistic $Z_{i}^{2}$ has a chi-square distribution with $p$ degrees of freedom, the percentage point of $Z_{i}^{2}$ can be obtained from a chi-square distribution. When the process has shifted to $\underline{\mu}$ from the target $\underline{\mu}_{0}, Z_{i}^{2}$ has a noncentral chi-square distribution with $p$ degrees of freedom and noncentrality parameter $\tau^{2}=n\left(\underline{\mu}-\underline{\mu}_{0}\right)^{\prime} \Sigma_{0}^{-1}\left(\underline{\mu}-\underline{\mu}_{0}\right)$.

The numerical performances of the matched FSI and two sampling interval VSI Shewhart charts based on $Z_{i}^{2}$ can be obtained by markov chain or integral equation approach.

\subsection{Multivariate EWMA Chart}

The EWMA control chart is a good alternative when we are interested in detecting small shifts. The performance of the EWMA control chart is approximately equivalent to that of the CUSUM chart, and in some ways, it is easier to implement and interpret than the CUSUM chart. In univariate control chart for mean of a variable, Lucas and Saccucci ${ }^{[9]}$ showed that the ARL's of EWMA are usually smaller than those of CUSUM up to a value of the shift near the one that the scheme was designed to detect for a normally distributed process. Beyond this shift, the ARL's of EWMA are larger than those of corresponding CUSUM.

A multivariate FSI EWMA control chart for the process mean vector $\underline{\mu}$ based on LRT statistic $Z_{i}^{2}(i=1,2, \ldots)$ in (2.2) is given by

$$
Y_{i}=(1-\lambda) Y_{i-1}+\lambda Z_{i}^{2},
$$

where $Y_{0}=\omega(\omega \geq 0)$ and $\lambda(0<\lambda \leq 1)$ is a smoothing constant. The statistic $Z_{i}^{2}$ is a function of the current sample $\underline{X}_{i}$, and $Y_{i}$ is the value of the EWMA after the observation at the $i$ th sample. This chart signals whenever $Y_{i} \geq h_{E}$.

For multivariate two sampling interval VSI EWMA chart based on $Z_{i}^{2}$, suppose the two sampling interval;

$d_{1}$ is used when $Z_{i}^{2} \in\left(g_{E}, h_{E}\right]$,

$d_{2}$ is used when $Z_{i}^{2} \in\left(0, g_{E}\right]$,

where $g_{E} \leq h_{E}$ and $d_{1}<d_{2}$. Since the distribution of control statistic $Z_{i}^{2}$ is known, the design parameters $g_{E}, h_{E}$ can be obtained to satisfy a specified ANSS and ATS by Markov chain approach or integral equation approach. And when the parameters are on-target or changed, the performances of the proposed EWMA charts can be evaluated by the Markov chain approach or integral equation approach. And $P$ (switch) and ANSW values of the VSI chart were obtained by simulation. 


\section{Conclusion}

The purpose of this paper is to investigate the performances and compare the proposed multivariate Shewhart and EWMA charts. In order to obtain the purposes, some kinds of standards for comparison are required both the process is in-control or out-of-control states. For simplicity in our computation for comparison, we assume that $\underline{\mu}_{0}=\underline{0}^{\prime}$, and all diagonal and offdiagonal components of $\Sigma_{0}$ are 1 and 0.3 , respectively. We put the sampling interva $d=1$ in FSI procedures and $(0.7,1.3)$ or $(0.1,1.9)$ in two sampling interval $\left(d_{1}, d_{2}\right)$ in VSI procedure. The numerical results were obtained when the ANSS and ATS of the in-control state was approximately equal to 370.4 , and $n=5$ for $p=2 \sim 5$. All numerical vaues of the Figures are obtained with the sampling intervals $d=1$ or $\left(d_{1}, d_{2}\right)=(0.1,1.9)$.

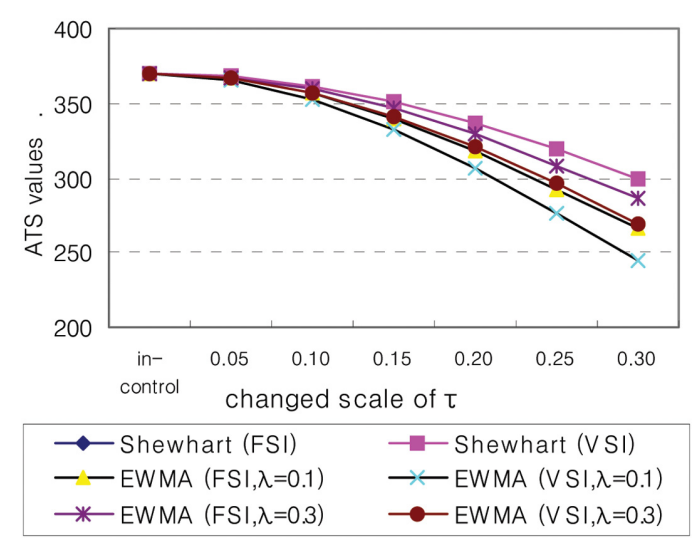

Fig. 1. ATS for small shifts $(p=3)$.

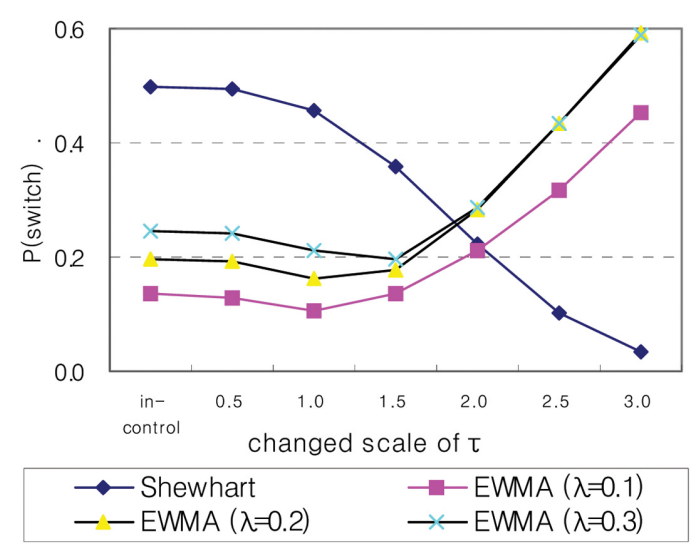

Fig. 2. $\mathrm{P}($ switch) values for $p=3$.

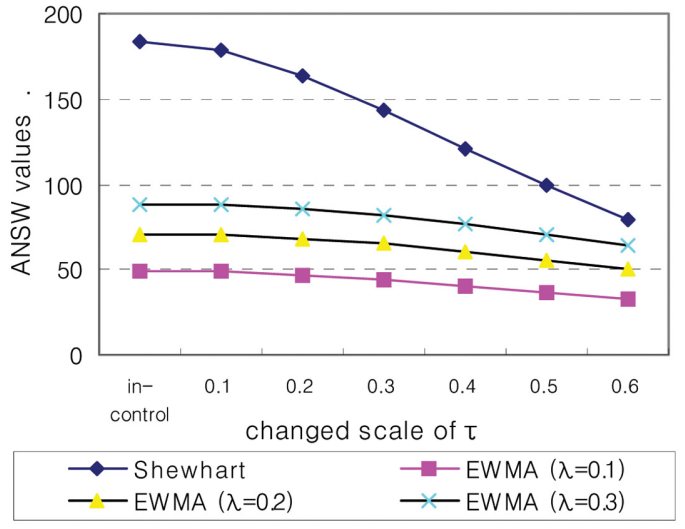

Fig. 3. ANSW values for $p=2$.

The design parameters $h_{E}$ and $g_{E}$ of the EWMA charts were calculated by Markov chain method with the number of transient states $r=100$. And the ANSS, ATS, and ANSW values of the EWMA chart when the process has changed were also obtained by Markov chain method or simulation with 10,000 runs. When $r$ is greater than 100 for various $p$, we found that asymptotic values using Markov chain method tends to be stabilized.

When small shifts in the process have occurred, the ATS and ANSW values of two sampling interval EWMA procedure with small smoothing constant is more efficient than the other proposed procedures. And when large shifts in the process have occurred, Tables show that the ATS of two sampling interval Shewhart procedure is more efficient than the other proposed EWMA procedures. And we also found that small values of smoothing constant $\lambda$ are more effective in detecting small or moderate changes in multivariate EWMA chart.

From the results of Tables and Figures for various $p$, two sampling interval charts are efficient for the corresponding FSI charts, and two sampling interval chart with $\left(d_{1}, d_{2}\right)=(0.1,1.9)$ is more efficient than $\left(d_{1}, d_{2}\right)=$ $(0.7,1.3)$ chart for small or moderate changes.

We also found that the EWMA procedure exhibits far fewer switches when compared to the Shewhart procedure. Also, it was established that smaller values of the parameter $\lambda$ for EWMA procedure will reduce the ANSW between two sampling intervals, respectively. And, We can notice that the optimal selection of the smoothing constant $\lambda$ in EWMA procedure mainly depends on the size of the shifts to be detected quickly. 


\section{References}

[1] S. W. Roberts, "Control Chart Tests Based on Geometric Moving Averages", Technometrics, Vol. 1, pp. 239-250, 1959.

[2] J. C. Arnold, "A Markovian Sampling Policy Applied to Quality Monitoring of Streams", Biomerics, Vol. 26, pp. 739-747, 1970.

[3] S. C. Smeach and R. W. Jernigan, "Further Aspect of a Markovian Sampling Policy for Water Quality Monitoring”, Biometrics, Vol. 33, pp. 41-46, 1977.

[4] M. R. Jr. Reynolds and J. C. Arnold, "Optimal OneSided Shewhart Control Charts with Variable Sampling Intervals between Samples", Sequential Analysis, Vol. 8, pp. 51-77, 1989.

[5] R. W. Amin, "Variable Sampling Interval Control Charts", Ph. D. Dissertation. Virginia Polytechnic
Institute and State University, Blacksberg, Virginia, 1987.

[6] M. R., Jr. Reynolds, R. W. Amin, and J. C. Arnold, "CUSUM charts with Variable Sampling Intervals", Technometrics, Vol. 32, pp. 371-384., 1990.

[7] V. C. C. Vargas, L. F. D. Lopes, and A. M. Sauza, "Comparative study of the performance of the CuSum and EWMA control charts", Comput. Ind. Eng., Vol. 46, pp. 707-724, 2004.

[8] C. Zou and F. Tsung, "Likelihood Ratio-Based Distrbution-Free EWMA Control Charts", J. Qual. Technol., Vol. 42, pp. 174-195, 2010.

[9] J. M. Lucas and M. S. Saccucci, "Exponentially Weighted Moving Average Control Schemes : Properties and Enhancements", Technometrics, Vol.32, pp. 1-12. 1990. 